\title{
A Framework for Integrating Intelligent Sensor Measurement Data into En- gineering Education
}

\author{
Dr. David O. Olowokere P.E., Texas Southern University \\ Dr. Abayomi Ajofoyinbo Ph.D, Texas Southern University
}

Biography - First Author: Dr. Olowokere received his PhD degree from the State University of New York in Buffalo NY, and he currently heads the engineering programs at Texas Southern University, Houston, Texas (TSU); and he also directs the TSU Aviation and Flight programs. In addition, he has been the principal investigator for a University-Industry partnership providing engineering support for Safety and Mission Assurance Program at the Johnson Space Center in Houston, and the NASA Marshall Center in Huntsville Alabama. He had served as principal investigator for research grants from several organizations including U.S. National Science Foundation, NASA, U.S. Department of Energy and the U.S. Department of Defense. Dr. Olowokere had previously been a faculty at the University of Alabama, University of Detroit, Wayne State University in Detroit and Bucknell University in Lewisburg Pennsylvania. His publications have appeared in several peer-reviewed journals including the ASCE Journal, Journal of Computer and Structures, Journal of Constructional Steel Research, and he has made presentations in numerous conferences, workshops and symposia worldwide in the general area of renewable energy technology, engineering practice and engineering management.

Biography - Second Author: Dr. Abayomi Ajofoyinbo received his PhD degree in Systems Engineering from the University of Lagos, Nigeria, in 2008. Dr. Ajofoyinbo is a Visiting Assistant Professor in the Department of Engineering at the Texas Southern University (TSU), Houston, Texas, USA. He is currently in-charge of Assessment for the engineering programs at TSU, and teaches courses in electrical/electronic and computer engineering. Between September 2013 and August 2014, Dr. Ajofoyinbo worked for TSU as Postdoctoral Fellow at the NSF CREST Centre for Research on Complex Networks. Dr. Ajofoyinbo is a Senior Lecturer in the Department of Systems Engineering, Faculty of Engineering, at the University of Lagos, Nigeria. His research interests are: intelligent control, embedded systems, wireless communications, wireless sensor networks, and engineering systems modeling \& analysis. He has published peer-reviewed papers in referred journals and international conferences. 


\title{
A Framework for Integrating Intelligent Sensor Measurement Data into Engineering Education
}

\author{
David O. Olowokere ${ }^{1}$ and Abayomi Ajofoyinbo ${ }^{2}$ \\ ${ }^{1,2}$ Department of Engineering \\ Texas Southern University, Houston, Texas, USA \\ Email: olowokeredo@tsu.edu ; ajofoyinboa@tsu.edu ${ }^{2}$;
}

\begin{abstract}
In the recent literature, researchers have investigated the mismatch between teaching and learning styles with different research objectives. This paper presents a framework for integrating intelligent sensor real-time measurement data into engineering education for innovative practice-oriented learning environment. In this integration framework, intelligent sensors are deployed on Local Area Networks (LAN) in engineering laboratories to measure physical quantities that may be used for classroom training. Engineering laboratories may involve measurement of dynamic processes since physical quantities measured by sensors such as temperature, humidity, pressure, displacement, voltage, current, etc., are continuous in nature. To improve the quality of measurement data there is a need to remove unwanted signals associated with the measured input signal; hence self-compensation algorithms are formulated for implementation in the sensor nodes. It is noted that presentations that use both visual and auditory styles reinforce learning for most students. Indeed, students' learning may be motivated and students' engagement and comprehension of fundamental engineering principles (or concepts) may be increased by a teaching style that balances concrete information with theoretical concepts. In the integration framework presented in this paper, professors/instructors may activate and take real-time reading from sensors located in the laboratories for purpose of illustrating or emphasizing engineering concepts (or principles). Moreover, in order to assess the acceptability of this framework in a learning environment, Questionnaires are administered to gather pertinent information from engineering professors and students. It is shown by the results obtained that this framework has high level of acceptability, and will create better learning experience for engineering students.
\end{abstract}

Keywords: Framework, integrating, wireless sensors, engineering education. 


\section{Introduction}

Presentations that use both visual and auditory styles reinforce learning for all students as visual learners remember best what they see while auditory learners remember much of what they hear, and then say (Felder and Silverman ${ }^{13}$ ). To teach both visual and auditory learners therefore, engineering professors should present information through lectures and reinforce such presentations with real-time demonstration in classroom. Students' learning may be motivated and students' engagement and comprehension of fundamental engineering principles may be increased by a teaching style that balances concrete information with theoretical concepts. It is noted that engineering laboratories may involve the measurement of dynamic processes, since physical quantities measured by sensors such as temperature, humidity, pressure, displacement, voltage, current, etc., are continuous in nature. In the recent literature, researchers have investigated the mismatch between teaching and learning styles with different research objectives. For example, Vasquez ${ }^{3}$ presented the development and implementation of an educational low-cost device/tool that can be set-up and used by students in and out of their engineering classes to assist their learning. In an interesting contribution, Xian and Madhavan ${ }^{8}$ presented the implementation of a middleware framework in the application server and a client on iPhone to facilitate access to sensor data. The output signal of a sensor may become "corrupted" with unwanted signals that prevent the actual useful signal required from being measured correctly. To effectively incorporate sensor measurement data into engineering learning environment, there is a need to ensure unwanted signals are removed, thereby improving quality of measurement data. It is noted that intelligent sensors possess higher processing capability and memory. The growing interest in the development of intelligent sensors is driven by a fundamental desire to get best quality measurement data. With respect to intelligent sensors capable of self-compensating of sensor measurements, Wang and Yan ${ }^{2}$ presented the mathematical modeling of strip electrostatic sensors for rotational speed measurement and associated experimental studies for the validation of the modeling results. In an interesting contribution, Petra et $\mathrm{al}^{5}$ developed mathematical model for resonant opto-thermoacoustic detection sensor which used sensed trace gas to convert optical radiation to heat which is detected due to the induced thermo-elastic deformation of a quartz tuning fork. The paper of Rivera-Mejia et $\mathrm{al}^{4}$ presented a methodology based on compensation methods to build reconfigurable measurement systems. The quantifiable comparison of several popular methods was shown to illustrate the selection of a computationally efficient compensation method. Other researchers have investigated virtual laboratories in engineering education wherein data acquisition interfaces are virtualized using the LabView software over the Internet platform. For example, Lin et al ${ }^{l}$ shows that remote distributed virtual laboratory cannot only save input in the experimental education but can also improve teaching level and promote students' learning autonomy. Fernandez-Sanchez et $\mathrm{al}^{6}$ described a computer-based system of improving engineering learning of complex technologies. Moreover, Burian et $\mathrm{al}^{l 1}$ described the development of resources for a virtual laboratory website for use in water resources engineering courses.

The current paper presents a framework for integrating sensor real-time measurement data into engineering education for innovative practice-oriented learning environment. The framework does not require Internet platform for its operations, since it is deployed on the Local Area Network (LAN). The rest of this paper is organized as follows. Motivation, systems modeling and design are discussed in Section 2. Results of numerical analysis are presented in Section 3. 
Discussion of results and contribution(s) of the research are presented in Section 4. Section 5 concludes the paper.

\section{Systems modeling and design}

This Section discusses motivation for the research in Section 2.1 and systems modeling and design in Section 2.2.

\subsection{Motivation for the research}

The research is motivated by the need to provide improved learning environment for engineering students, whereby professors/instructors can access the laboratory to read real-time data to illustrate or explain concepts (or principles) during classroom session.

\subsection{Systems modeling and design}

From the perspective of engineering education, the current paper is related to systems engineering as it creates enabling framework for integrating the different entities (or subsystems) involved in teaching and learning. Such integration will produce better learning experience for the students (Fig. 1). It is expected that the integration framework presented in this paper will further narrow the gap between practical and theoretical knowledge acquisition by engineering students.

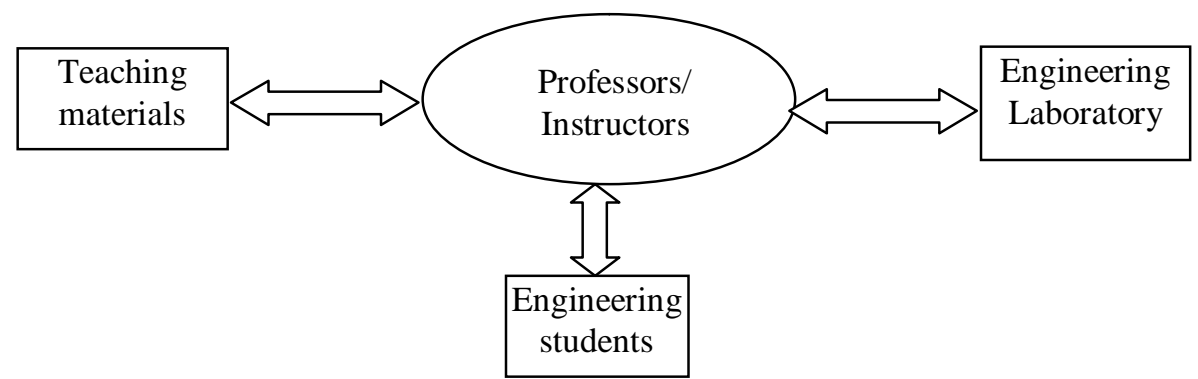

Fig. 1. Conceptual framework of the integrated learning environment

\section{(i) Integrating sensor measurement data into engineering education}

The schematic diagram for integrating sensor real-time measurement data into engineering education is presented in Fig 2. In this framework, the connection of the wireless sensors to the LAN is achieved using a proxy-based approach (Neves and Rodrigue ${ }^{9}$; Rodrigues and Neves ${ }^{10}$ ). In this approach, wireless sensor nodes communicate through dedicated wireless sensor network (WSN) protocols, while the sink node acts as a Proxy to the LAN converting IP (Internet Protocol) to/from the dedicated WSN protocol. The transformation can be performed at the application level (Mayer and Fritsche ${ }^{12}$ ). At any time during classroom lecture sessions, professors/instructors can access real-time sensor measurement data to illustrate (or explain) engineering principles (or concepts). In this framework, the sink node queries the wireless 
sensor network using dedicated protocol, and connects to the LAN using the IP protocol. Moreover, the IP cameras deployed on the LAN and located in engineering laboratories will provide real-time view of the laboratories when activated by professors/instructors during classroom lecture session.

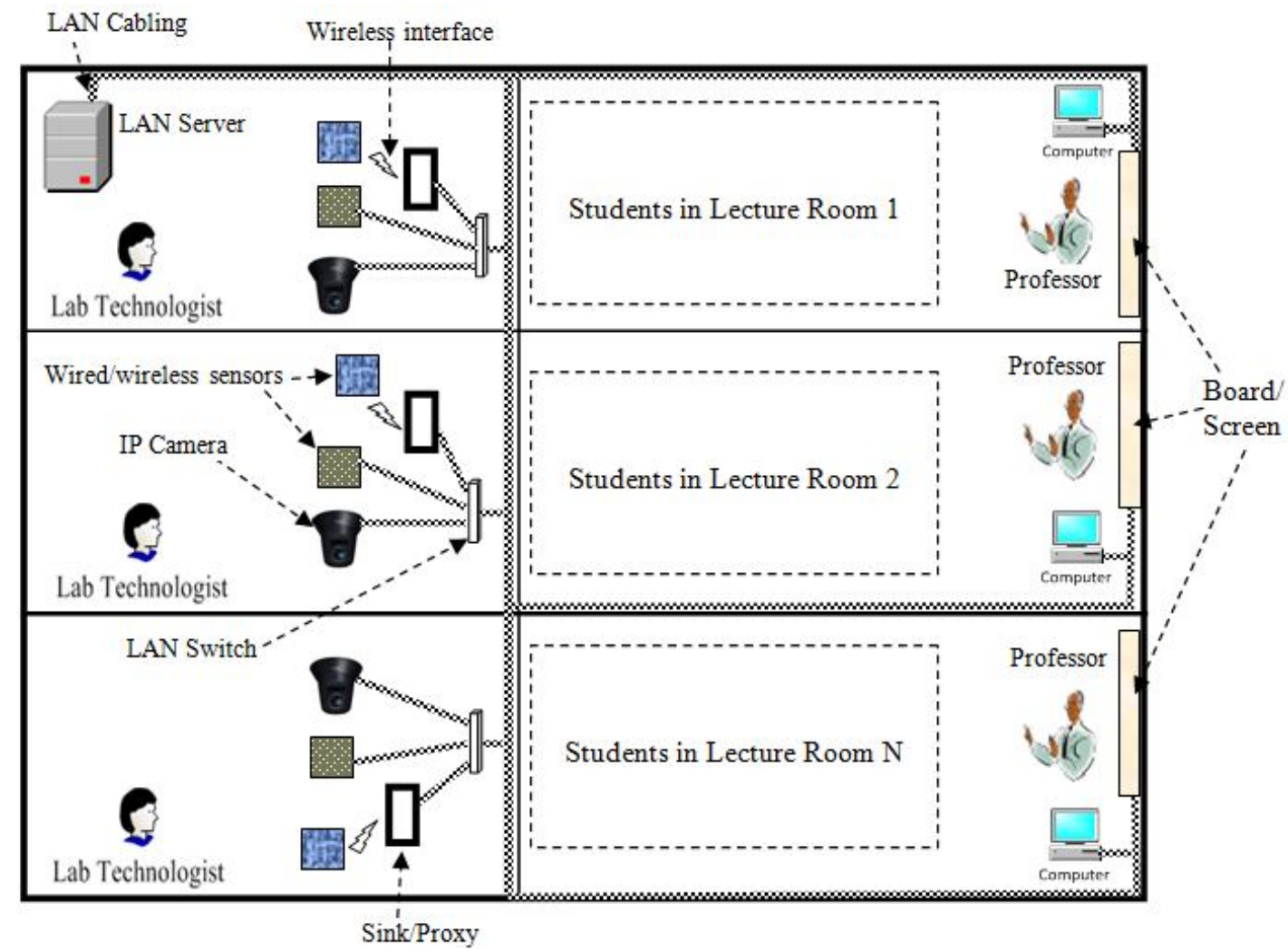

Fig. 2. Schematic diagram of the integration framework

To assess the acceptability of this new framework for integrating intelligent sensor real-time measurement data into engineering education, well-structured Questionnaires are administered to collect pertinent information from engineering professors and students. For this exercise, fortyfive (45) engineering students are presented with six (6) statements and asked to show their level of agreement (or disagreement) with each statement by assigning a number between 1 and 5 , defined as follows:
1: I strongly agree
2: I agree
3: I do not know (Indifferent)
4: I disagree
5: I strongly disagree

The students' survey statements are as follow:

1. The integration framework for teaching and learning presented in this paper may improve students' understanding of engineering concepts (or principles) during lectures.

2. Lecture time may be wasted by this integration framework and it may also make lectures uninteresting to students. 
3. Using the framework may be intellectually stimulating as students will be able to directly ask the professor practical and pertinent questions during lecture based on the equipment (or materials) in the Laboratory.

4. Teaching approach whereby professor can connect to the Laboratory during lecture and take sensor measurement data for purpose of explaining (or emphasizing) engineering concepts (or principles) is significant in engineering education.

5. The teaching approach may significantly reduce existing mismatch between learning and teaching styles in engineering courses.

6. The framework presented in this paper may be implemented in engineering departments of colleges/universities.

Moreover, eight (8) professors/instructors are presented with six (6) statements and asked to show their level of agreement (or disagreement) with each statement by assigning a number between 1 and 5 defined as follows:

1: I strongly agree

2: I agree

3: I do not know (Indifferent)

4: I disagree

5: I strongly disagree

The professors/instructors' survey statements are as follow:

1. The integration framework presented in this paper in which professor can connect to the Laboratory during classroom-lecture to take sensor measurement data and use such data to explain (or emphasize) engineering concepts (or principles) may improve quality of lecture and reinforce students' learning.

2. Using this framework may waste lecture-time, make lecture uninteresting to professor/instructor, and indeed may not be intellectually stimulating to students.

3. This framework will enable professor emphasize concrete information during lectures and create better learning experience for students.

4. This framework may reduce existing mismatch between learning and teaching styles in engineering courses as professor will be able to impart knowledge in students beyond the theory taught in classroom.

5. The teaching style presented in this framework is significant in engineering education as it may create a practice-oriented learning environment.

6. This framework may be implemented in engineering departments of colleges/universities.

\section{(ii) Self-compensation of sensor measurements}

To ensure that data used for classroom demonstration of engineering concepts (or principles) realistically represent measured phenomenon, there is a need to deploy sensors that have capability for self-compensation of measurement data. In this paper for example, wired/wireless sensor that incorporates (a) one energy storage component (e.g., temperature/pressure sensors), and (b) two energy storage components (e.g., accelerometers) are considered. To improve the quality of measurement data therefore, self-compensation algorithm(s) may be implemented in 
sensor nodes deployed in engineering laboratories. Table 1 below shows the notations used in modeling self-compensation in sensor nodes.

Table 1:Notations

\begin{tabular}{|l|l|}
\hline Variable & Description \\
\hline $\mathrm{u}(\mathrm{t})$ & Real sensed quantity (real inputs) \\
\hline $\mathrm{n}(\mathrm{t})$ & Noise (unwanted input) \\
\hline $\mathrm{y}(\mathrm{t})$ & Intermediate output of sensor node \\
\hline $\mathrm{z}(\mathrm{t})$ & Final output of wireless sensor node \\
\hline
\end{tabular}

The schematic diagram of the sensing subsystem showing relationships between measured input, noise (i.e., unwanted signals), intermediate output and final output of a sensor node is provided in Fig. 3 below.

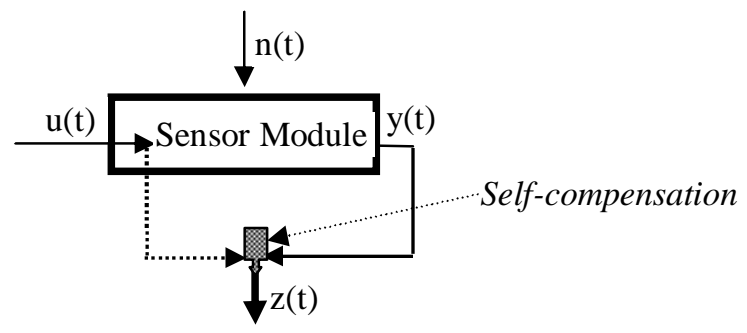

Fig. 3. Schematic diagram of sensing subsystem showing measured input, noise and sensor outputs.

The relationships between the total input $f(t)$ and intermediate output $y(t)$ of a wireless sensor node are modeled for sensors with one storage component and sensors with two storage components as follows:

a) For wireless sensor node with one storage component:

$$
\frac{d y(t)}{d t}+\beta y(t)=f(t)
$$

where $f(t)=u(t)+n(t) ; t>0$; and $y(0)=0$.

Upon taking the Laplace transform and using the initial condition $y(0)=0$, Eq. (1) yields,

$$
Y(s)=F(s) \cdot \frac{1}{(s+\beta)} .
$$

Using the convolution theorem $\left(\operatorname{Logan}^{7}\right)$, the inverse Laplace transform of Eq. (2) is subsequently obtained as

$$
y(t)=\int_{0}^{t} f(\tau) \cdot e^{-\beta(t-\tau)} d \tau
$$


b) For wireless sensor node with two storage components:

$$
\frac{d^{2} y(t)}{d t^{2}}+\beta_{1} \frac{d y(t)}{d t}+\beta_{2} y(t)=f(t) .
$$

where $f(t)=u(t)+n(t) ; t>0 ; y^{\prime}(0)=0$; and $y(0)=0$.

Upon taking the Laplace transform and using initial conditions $y^{\prime}(0)=0$ and $y(0)=0$, Eq. (4) yields,

$$
Y(s)=F(s) \cdot\left[\frac{1}{\left(s+\frac{\beta_{1}}{2}\right)^{2}}-\frac{1}{\left(\left(\frac{\beta_{1}}{2}\right)^{2}+\beta_{2}\right)}\right] .
$$

Using the convolution theorem, the inverse Laplace transform of Eq. (5) gives

$$
y(t)=\int_{0}^{t} f(\tau) \cdot\left((t-\tau) e^{-\frac{\beta_{1}}{2}(t-\tau)}-\frac{4}{\beta_{1}^{2}+4 \beta_{2}}\right) d \tau .
$$

For example, let input signals to the sensing subsystem be sinusoidal inputs defined as

$$
u(t)=3 \sin \left(\frac{\pi t}{4}\right)
$$

and

$$
n(t)=\frac{3}{5} \sin \left(\frac{3 \pi t}{4}\right)
$$

Upon substituting the values of $u(t)$ and $n(t)$ in Eq. (3) above, Eq. (9) is obtained as

$$
y(t)=3 e^{-\beta t}\left[\begin{array}{l}
\left(\frac{4}{\pi^{2}+4 \beta^{2}}\right) \cdot e^{\beta t}\left(\beta \sin \left(\frac{\pi t}{4}\right)-\frac{\pi}{4} \cos \left(\frac{\pi t}{4}\right)\right)+\left(\frac{16}{45 \pi^{2}+8}\right) \cdot e^{\beta_{t}}\left(\beta \sin \left(\frac{3 \pi t}{4}\right)-\left(\frac{3 \pi}{4} \cos \left(\frac{3 \pi t}{4}\right)\right)\right) \\
+\left(\frac{\pi}{\pi^{2}+4 \beta^{2}}\right)+\left(\frac{12 \pi}{45 \pi^{2}+80}\right)
\end{array}\right]
$$


From the foregoing, the self-compensation of measurement data to recover the real measured quantity is consequently achieved by

$$
z(t)=y(t)-n(t)
$$

where $z(t)$ is the resultant sensor output (with no unwanted input). These self-compensating algorithms may be implemented in sensor nodes deployed in the engineering laboratories.

\section{Results of numerical analysis}

(i) Average survey responses to Questionnaires

Tables 1 and 2 below show the average survey responses of students and professors/instructors respectively.

Table 1: Students' survey responses

\begin{tabular}{|l|l|c|}
\hline S/N & \begin{tabular}{l} 
Survey statements \\
\hline 1
\end{tabular} & $\begin{array}{l}\text { Average scores } \\
\text { (45 students) }\end{array}$ \\
\hline 2 & $\begin{array}{l}\text { The integration framework for teaching and } \\
\text { learning presented in this paper may improve } \\
\text { (or principles) during lectures. }\end{array}$ & $\begin{array}{l}\text { Lecture time may be wasted by this integration } \\
\text { framework and it may also make lectures } \\
\text { uninteresting to students. }\end{array}$ \\
\hline 3 & $\begin{array}{l}\text { Using the framework may be intellectually } \\
\text { stimulating as students will be able to directly ask } \\
\text { the professor practical and pertinent questions } \\
\text { during lecture based on the equipment (or } \\
\text { materials) in the Laboratory. }\end{array}$ & 2.1 \\
\hline 4 & $\begin{array}{l}\text { Teaching approach whereby professor can connect } \\
\text { to the Laboratory during lecture and take sensor } \\
\text { measurement data for purpose of explaining (or } \\
\text { emphasizing) engineering concepts (or principles) } \\
\text { is significant in engineering education. }\end{array}$ & 2.0 \\
\hline 5 & $\begin{array}{l}\text { The teaching approach may significantly reduce } \\
\text { existing mismatch between learning and teaching } \\
\text { styles in engineering courses. }\end{array}$ & $\begin{array}{l}\text { The framework presented in this paper may be } \\
\text { implemented in engineering departments of } \\
\text { colleges/universities. }\end{array}$ \\
\hline 6
\end{tabular}

Table 2: Professors/Instructors' survey responses

\begin{tabular}{|l|l|c|}
\hline S/N & Survey statements & $\begin{array}{c}\text { Average scores } \\
\text { (8 professors) }\end{array}$ \\
\hline 1 & $\begin{array}{l}\text { The integration framework presented in this paper } \\
\text { in which professor can connect to the Lab during } \\
\text { classroom-lecture to take sensor measurement data } \\
\text { and use such data to explain (or emphasize) } \\
\text { engineering concepts (or principles) may improve } \\
\text { quality of lecture and reinforce students' learning. }\end{array}$ & 1.9 \\
\hline
\end{tabular}




\begin{tabular}{|l|l|c|}
\hline 2 & $\begin{array}{l}\text { Using this framework may waste lecture-time, } \\
\text { make lecture uninteresting to professor/instructor, } \\
\text { and indeed may not be intellectually stimulating to } \\
\text { students. }\end{array}$ & 4.1 \\
\hline 3 & $\begin{array}{l}\text { This framework will enable professor emphasize } \\
\text { concrete information during lectures and create } \\
\text { better learning experience for students }\end{array}$ & 1.6 \\
\hline 4 & $\begin{array}{l}\text { This framework may reduce existing mismatch } \\
\text { between learning and teaching styles in } \\
\text { engineering courses as professor will be able to } \\
\text { impart knowledge in students beyond the theory } \\
\text { taught in classroom. }\end{array}$ & 2.0 \\
\hline 5 & $\begin{array}{l}\text { The teaching style presented in this framework is } \\
\text { significant in engineering education as it may } \\
\text { create a practice-oriented learning environment. }\end{array}$ & 1.4 \\
\hline 6 & $\begin{array}{l}\text { This framework should be implemented in } \\
\text { engineering departments of colleges/universities. }\end{array}$ & 1.9 \\
\hline
\end{tabular}

(ii) Graphical presentations of survey results

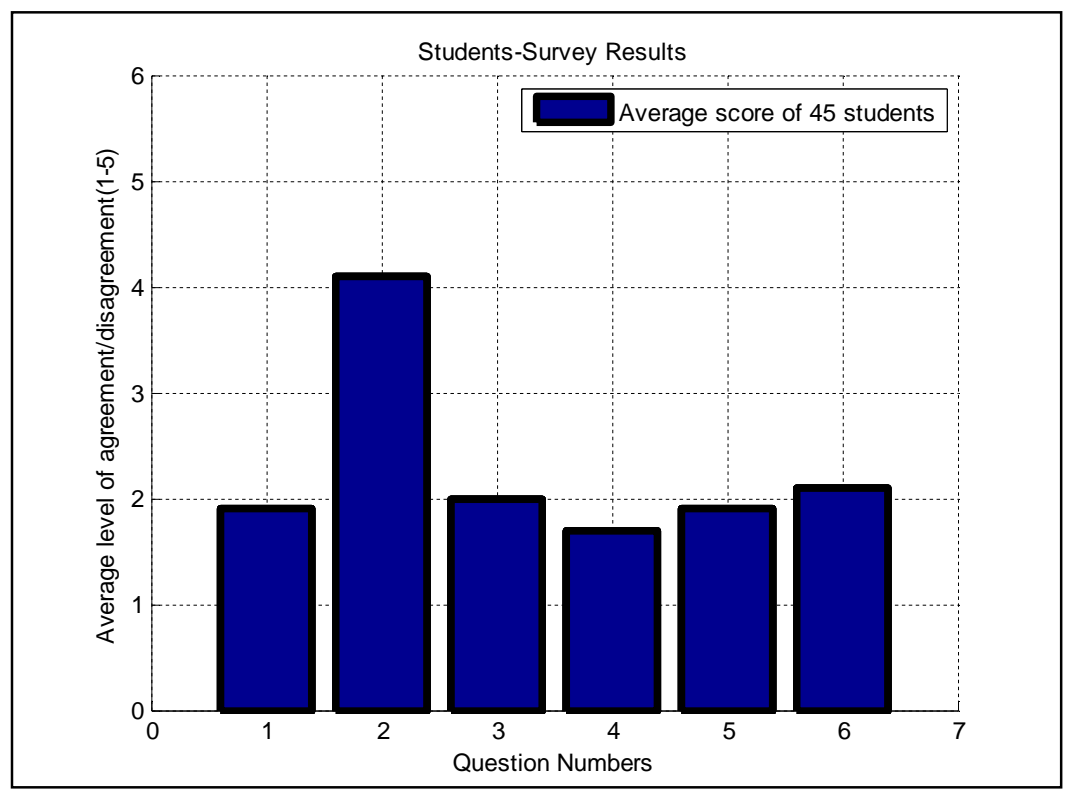

Figure 4. Students' survey results (average score)

\section{Discussion of results and contribution of research}

\subsection{Discussion of results}

The average scores in respect of survey responses to the Questionnaires are presented graphically in Figures 4 and 5 for the students' and professors' surveys respectively. The implications of the average scores are discussed below. 
(a) Students' survey: average scores

Statement \#1: 95\% agreed with this statement while 5\% either strongly agree with this statement or are indifferent.

Statement \#2: $82 \%$ disagreed with this statement while $18 \%$ either strongly disagree with this statement or are indifferent.

Statement \#3: $100 \%$ agreed with this statement.

Statement \#4: $85 \%$ agreed with this statement while 15\% either strongly agree with this statement or are indifferent.

Statement \#5: 95\% agreed with this statement while 5\% either strongly agree with this statement or are indifferent.

Statement \#6: $70 \%$ agreed with this statement while 30\% either strongly agree with this statement or are indifferent or disagreed with this statement.

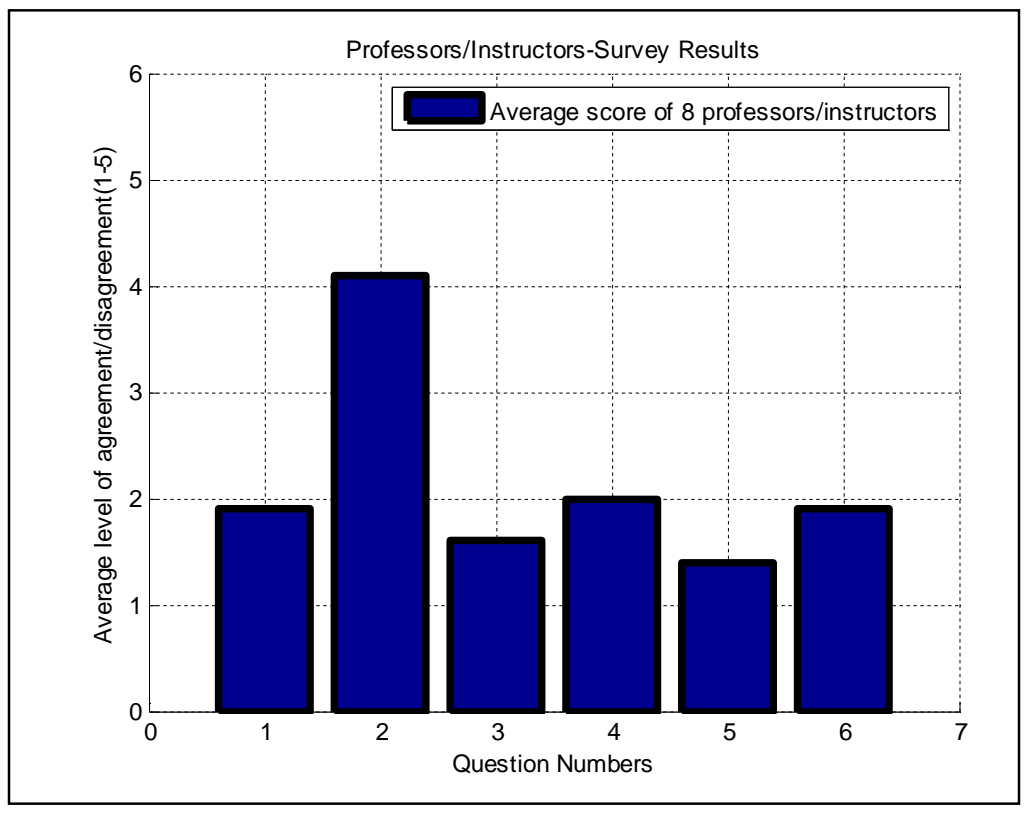

Fig. 5. Professors/Instructors' survey results (average score)

(b) Professors' survey: average scores

Statement \#1: 95\% agreed with this statement while 5\% either strongly agree with this statement or are indifferent.

Statement \#2: $82 \%$ disagreed with this statement while $18 \%$ either strongly disagree with this statement or are indifferent.

Statement \#3: 80\% agreed with this statement while 20\% either strongly agree with this statement or are indifferent.

Statement \#4: $100 \%$ agreed with this statement.

Statement \#5: 70\% agreed with this statement while 30\% either strongly agree with this statement or are indifferent.

Statement \#6: 95\% agreed with this statement while 5\% either strongly agree with this statement or are indifferent or disagreed with this statement. 


\subsection{Contribution of research}

This research contributes to knowledge by providing a framework for integrating sensor realtime measurement data into engineering education, thereby creating an innovative practiceoriented learning environment for engineering students.

\section{Conclusion}

In this paper, the integration of sensors real-time measurement data into engineering education to motivate students' learning was investigated. This paper presented a framework for integrating intelligent wired/wireless sensors real-time measurement data into engineering education for innovative practice-oriented learning environment. In this integration model, intelligent wired/wireless sensors deployed on the Local Area Networks (LAN) in engineering laboratories measure physical quantities. Self-compensation algorithms were formulated for implementation in these intelligent sensor nodes, thereby providing capabilities for self-compensating of measurement data. During classroom sessions, professors/instructors could connect to sensors located in engineering laboratories and use real-time measurement data for illustrations. In order to assess the acceptability of this framework in a learning environment, Questionnaires were administered to gather pertinent information from engineering professors/instructors and students. The results show that the integration framework presented in this paper has high level of acceptability, will reduce existing mismatch in teaching and learning styles, and provide improved learning experience for engineering students.

\section{Acknowledgment}

This research is supported in part by the National Science Foundation (NSF) under Grants: NSF-1137732 and NSF-1241626 (Center for Research Excellence in Science and Technology CREST).

\section{References}

[1] Lin Y., Lv C., Li Y; The description and analysis of remote network virtual laboratory. International Journal of Multimedia and Ubiquitous Engineering; 2014, vol. 9, no. 9, pp. 163-170.

[2] Wang L., Yan Y.; Mathematical modeling and experimental validation of electrostatic sensors for rotational speed measurement. Measurement Science and Technology, 2014, vol. 25, 12pp.

[3] Vasquez H.; Integration of Sensors and Low-Cost Microcontrollers into the Undergraduate Mechanical Engineering Design Sequence. $120^{\text {th }}$ ASEE Annual Conference \& Exposition, June 23-26, 2013; Atlanta, Paper ID\# 6412.

[4] Rivera-Mejia, J., Carrillo-Romero, M., Herrera-Ruiz, G.; Self-compensation to build reconfigurable measurement systems. Instrumentation \& Measurement Magazine, IEEE, April 2013, vol.16, no.2, pp.10-19.

[5] Petra N., Zweck J., Minkoff S.E., Kosterev A. A., Doty J.H.; Modeling and design optimization of a resonant opto-thermoacoustic trace gas sensor. Society for Industrial and Applied Mathematics Journal of Applied Mathematics, 2011, vol. 71, no. 1, pp. 309-332. 
[6] Fernandez-sanchez P., Salaverria-Garnacho A., Valdes V.G., Mandado E. Using a virtual laboratory as selfassessment tool for complex technologies learning. $1^{\text {st }}$ World Engineering Education Flash Week, Lisbon, Portugal; 2011, pp. 569-573.

[7] Logan J.D.; A first course in differential equations; $2^{\text {nd }}$ Edition, 2011. Springer Science, USA

[8] Xian H., Madhavan K.; AC 2010-1857: Sensorworld: A New Approach to Incorporating Large-Scale Sensor Data into Engineering Learning Environments. American Society for Engineering Education (ASEE) Conference, 2010.

[9] Neves P.A.C.D.S., Rodrigues J.J.P.C.; Internet Protocol over wireless sensor networks, from myth to reality. Journal of communications, vol. 5, no. 3, March 2010.

[10] Rodrigues J.J.P.C., Neves P.A.C.D.S.; A survey on IP-based wireless sensor networks solutions. International Journal of Communication Systems; 2010, vol. 23, pp. 963-981.

[11] Burian S., Kalyanapu A., Houdeshel D., Judi D., Pomeroy C.; A web-based virtual laboratory for water resources engineering education. American Society of Engineering Education (ASEE) Conference, 2010; AC 2009-1446.

[12] Mayer K and Fritsche W.; IP-Enabled wireless sensor networks and their integrations into the internet. Proceedings of the First International Conference on Integrated Ad-hoc and Sensor Networks, Nice, France, 2006.

[13] Felder R.M., Silverman L.K.; Learning and Teaching Styles in Engineering Education. Engineering Education, vol. 78, issue no. 7, 1988, pp. 674-681. 